\title{
Effect of oxygen deficiency $(\delta)$ on transition temperature of yttrium cuprate superconductors
}

\author{
R K SINGH, DINESH VARSHNEY ${ }^{*+}$ and A K KHASKALAM \\ School of Physics, Guru Ghasidas University, Bilaspur 495009, India \\ ${ }^{+}$School of Physics, Devi Ahilya University, Khandwa Road, Indore 452001, India \\ MS received 9 May 1996
}

\begin{abstract}
The nature of pairing mechanism as well as transition temperature of yttrium cuprates is discussed using the strong coupling theory. An interaction potential has been developed for the layered structure with two conducting $\mathrm{CuO}_{2}(\mathrm{a}-\mathrm{b})$ layers in a unit cell. The interaction potential properly takes care of electron electron, electron-phonon and electronplasmon interactions. Furthermore, the electron phonon coupling parameter $(\lambda)$, the modified Coulomb repulsive parameter $\left(\mu^{*}\right)$ and the 2D acoustic phonon (plasmon) energy as a function of oxygen deficiency is worked out. Finally. the superconducting transition temperature $\left(T_{c}\right)$ is then evaluated by using these coupling parameters and obtained $T_{c}=95(92) \mathrm{K}$ for $\mathrm{Y}(\mathrm{Yb}) \mathrm{Ba}_{2} \mathrm{Cu}_{3} \mathrm{O}_{3}$, superconductors with $\delta=0 \cdot 0$. The model parameters estimated from the layered structure approach are consistent with the strong coupling theory. The result deduced on the variation of $T_{\mathrm{c}}$ with $\delta$ are in fair agreement with the earlier reported data on yttrium cuprates. The analysis of the above results are discussed.
\end{abstract}

Keywords. Oxygen deficiency: phonons; plasmons; coupling parameter; superconducting transition temperature.

\section{Introduction}

The discovery of superconductivity in the layered copper oxides with lanthanum as rare-earth $\left(T_{\mathrm{c}} \cong 40 \mathrm{~K}\right)$ has stimulated an extensive theoretical and experimental work (Bednorz and Muller 1986). Subsequently, the announcement of $\mathrm{YBa}_{2} \mathrm{Cu}_{3} \mathrm{O}_{7-\delta}$ as a superconductor with $T_{\mathrm{c}}=90 \mathrm{~K}$ (Wu et al 1987) has renewed the interest. Both the cuprates have in general a common feature of conducting $\mathrm{CuO}_{2}$ planes. The copper charge valence neutralities is perturbed either by doping concentration $(x)$ (e.g. in $\mathrm{La}_{2-x} \mathrm{Sr}_{x} \mathrm{CuO}_{4}$ ) or by oxygen deficiency $\left(\delta\right.$ ) (as in $\mathrm{YBa}_{2} \mathrm{Cu}_{3} \mathrm{O}_{7-\delta}$ ). The layered structure consists of strong superconducting planes and the transport is mostly conveyed along the strongly superconducting $\mathrm{CuO}_{2}(\mathrm{a}-\mathrm{b})$ planes. It is believed that the crystal structure and transport properties of $\mathrm{YBCO}$ are strongly dependent on the oxygen deficiency $(\delta)$. It may be noted that for the lower values of $\delta(=0.0)$ the phase is orthorhombic and becomes superconducting at low temperatures. Furthermore, for higher values of $\delta(=1 \cdot 0)$, the phase is tetragonal and non-superconducting at all temperatures. In fact the behaviour of transition temperature $\left(T_{c}\right)$ with oxygen deficiency is anomalous and has been noticed since their discovery. It is therefore necessary to understand the behaviour of $T_{\mathrm{c}}$ with $\delta$ from the point of view of theory.

The yttrium cuprates contain two-dimensional (2D) conducting $\mathrm{CuO}_{2}$ planes as well as one-dimensional (1D) $\mathrm{CuO}$ chains. The role of chains in the conduction mechanism is not yet clearly understood. Cava et al (1990) and Kerkels et al (1992) have reported the behaviour of transition temperature with oxygen deficiency $(\delta)$ in $\mathrm{YBa}_{2} \mathrm{Cu}_{3} \mathrm{O}_{7-\delta}$

\footnotetext{
*For correspondence
} 
superconductors. It is observed that $T_{\mathrm{c}}$ varies smoothly showing two plateaus with $\delta$ in the range $0.0<\delta<1.0$. Furthermore, the $T_{\mathrm{c}}$ varies stepwise from near $90 \mathrm{~K}$ $[0.0<\delta<0.2]$ and decreases rapidly to about $60 \mathrm{~K}[0.2<\delta<0.3]$. It is nearly constant in the range $0.3<\delta<0.5$ and then drops sharply from $60 \mathrm{~K}$ to $0 \mathrm{~K}$ for $0.5<\delta<0.6$. The first plateau is observed at about $90 \mathrm{~K}$ for an oxygen content 0.0 to 0.2 and a second plateau is present at $60 \mathrm{~K}$ over the oxygen deficiency smaller than 0.6 . It is understood that in yttrium cuprates, the superconductivity is sensitive to oxygen deficiency $(\delta)$. Kerkels et al (1992) have extensively studied the effect of substitution of Y ion by Sm and $\mathrm{Yb}$ ions in these cuprates. Similar results on the behaviour of $T_{\mathrm{c}}$ with $\delta$ have been observed as those of $Y$ doped superconductors with a negligible change in maximum superconducting $T_{\mathrm{c}}$ at $\delta=0 \cdot 0$.

With regard to pairing mechanism it may be noted that it is extremely difficult to achieve a transition temperature of $40 \mathrm{~K}$ or more based on the conventional electronphonon interactions. Over the last several years the plasmon mechanism emerged in the central theme which is not only important in the additional pairing but could also lead to such high $T_{\mathrm{c}}$ values. Earlier Jha (1987) emphasized the role of plasmons in YBCO superconductors and explained the high $T_{\mathrm{c}}$. Later on, Tewari and Gumber $(1990)$ predicted the high $T_{c}$ and the anisotropic properties in the layered structure YBCO systems. They pointed out that the plasmons were able to form an additional pairing mechanism in yttrium copper oxide superconductors. Earlier, Singh et al (1993) investigated the effects of two dimensional (2D) acoustic plasmons and succeeded in predicting the composition dependence of the transition temperature in $\mathrm{La}_{2-x}(\mathrm{Ba}, \mathrm{Sr})_{x} \mathrm{CuO}_{4}$ superconductors. Besides these theoretical predictions, the plasmons were observed by electron-energy loss spectroscopy in $\mathrm{YBa}_{2} \mathrm{Cu}_{3} \mathrm{O}_{7-\delta}$ superconductors (Bozovic 1990). Recently Varshney and Singh (1995) devoted their effort to develop a mechanism (joint phononplasmon) based on free electron layered electron gas (FELEG) model of quasi two dimensional layers in lanthanum cuprates with single $\mathrm{CuO}_{2}$ layer. The approach facilitates the dielectric function and dispersion relations of phonon and plasmon modes. It was noticed that joint phonon-plasmon mechanism with properly incorporated layered structure in the interaction potential not only described the pairing mechanism but also explained consistently the superconducting state parameters too. Motivated from the experimental observations (Cava et al 1990; Kerkels et al 1992) and earlier theoretical investigations (Jha 1987; Tewari and Gumber 1990; Singh et al 1993; Varshney and Singh 1995), we thought it pertinent to look for the pairing mechanism and behaviour of transition temperature with oxygen deficiency $(\delta)$ in yttrium cuprates. In the present study, we have extended the earlier approach (Singh et al 1993; Varshney and Singh 1995) by considering two conducting (2D) $\mathrm{CuO}_{2}$ planes in a unit cell. The main feature of the developed approach is to estimate the electronphonon coupling parameter $(\lambda)$, the modified Coulomb repulsive parameter $\left(\mu^{*}\right)$ and the $2 \mathrm{D}$ acoustic phonon (plasmon) $h \omega_{-}\left(\hbar \omega_{+}\right)$energy to evaluate the superconducting transition temperature in yttrium cuprate as a function of oxygen deficiency. We have made an effort to understand the experimental observations on $T_{\mathrm{c}}$ with $\delta$ based on realistic parameters by a simple layered electron gas which properly incorporates the layered structure.

The plan of the present study is as follows. In $\S 2$ we develop the interaction potential by considering two conducting $2 \mathrm{D} \mathrm{CuO}_{2}$ layers in a unit cell separated by insulating layers. We work out the coupling parameters and the energy of the generated polarized waves along the $a-b$ plane. In the present study we have not considered the role of 


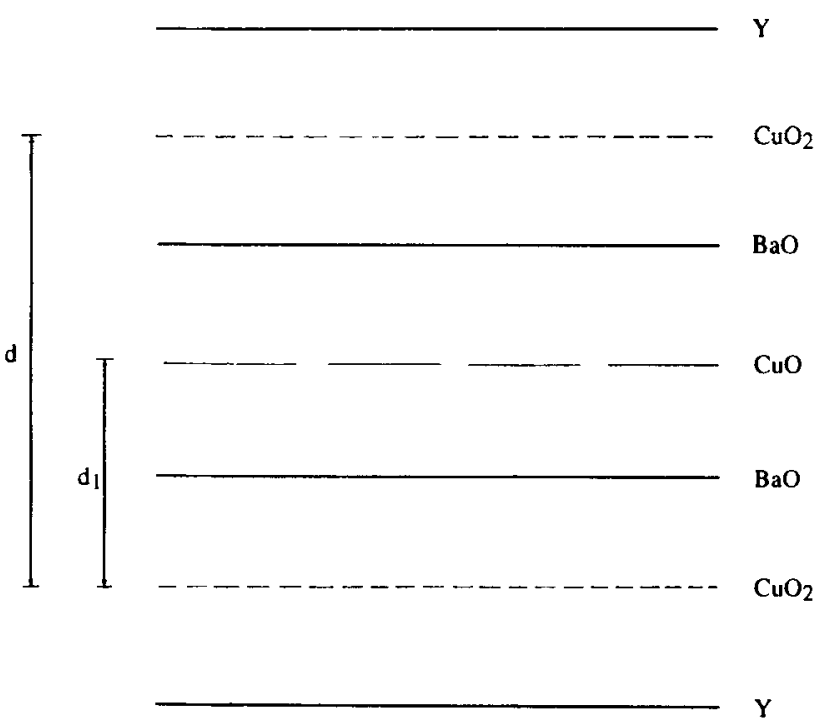

Figure 1. Layered structure of $\mathrm{YBa}_{2} \mathrm{Cu}_{3} \mathrm{O}_{7-\delta}$ superconductors.

chains due to the fact that they will act as a reservoir and their effects will be studied separately. From the deduced coupling parameters in the strong coupling theory the transition temperature as a function of oxygen deficiency is estimated. The results obtained from the simple approach are analysed and discussed in $\S 3$. Finally, $\S 4$ is devoted to conclusions.

\section{Theoretical formalism}

The change in oxygen deficiency $(\delta)$ in $\mathrm{ABa}_{2} \mathrm{Cu}_{3} \mathrm{O}_{7-\delta}(\mathrm{A}=\mathrm{Y}, \mathrm{Yb})$ superconductors is believed to introduce free charge carriers (holes) in the conducting $\mathrm{CuO}_{2}$ planes. These charge carriers interact by way of Coulomb potential with the nearby $\mathrm{CuO}_{2}$ planes. Consider a set of two dimensional (2D) layered electron gas where the charge carriers interact by polarized waves (quantized lattice vibrations and collective excitations). The layered structure of $\mathrm{YBa}_{2} \mathrm{Cu}_{3} \mathrm{O}_{7-\delta}$ is regarded as the stack of planes $\mathrm{Y}, \mathrm{CuO}_{2}$, $\mathrm{BaO}$, oxygen deficient $\mathrm{CuO}$ (chain), $\mathrm{CuO}_{2}, \mathrm{BaO}, \mathrm{CuO}_{2}, \mathrm{Y}$ (see figure 1). It is assumed that a unit cell consists of $2 \mathrm{D}$ layers in the $\mathrm{a}-\mathrm{b}$ plane with a relatively weak coupling between the planes in the z-direction. We made following assumptions: (i) there are two conducting $\mathrm{CuO}_{2}$ planes per unit cell, (ii) the $\mathrm{CuO}_{2}$ planes form an infinite array of planes along c-axis, (iii) the non-conducting planes between $\mathrm{CuO}_{2}$ planes are considered as an uniform dielectric medium with a background dielectric constant $\left(\varepsilon_{0}\right)$ and (iv) the oxygen deficient $\mathrm{CuO}$ chain stabilized the charge carriers in the conducting plane.

The effective interaction potential between charge carriers is expressed as

$$
V(q, \omega)=\frac{V_{c}(q)}{\varepsilon(q, \omega)}
$$

with $V_{c}(q)$ is the bare Coulomb potential, $\varepsilon(q, \omega)$ the longitudinal dielectric function for a single band of charge carriers and $q$ the wave vector along a-b plane. 
The Fourier transformed effective potential between the charge carriers is

$$
V\left(q, q_{z}, \omega\right)=-\frac{2 \pi e^{2}}{q \varepsilon_{0}} \frac{S\left(q, q_{z}\right)}{\varepsilon\left(q, q_{z}, \omega\right)}
$$

Here, $S\left(q, q_{z}\right)$ is the structure factor as

$$
S\left(q, q_{\mathrm{z}}\right)=\frac{\sin h(q d)}{\cos h(q d)-\cos \left(q_{\mathrm{z}} d\right)},
$$

with $d$ as the distance between the two $\mathrm{CuO}_{2}$ planes in a unit cell, $q_{\mathrm{z}}$ the wave vector in the perpendicular direction and $\varepsilon_{0}$ denotes the background dielectric constant of the surrounding materials. The longitudinal dielectric response function $\varepsilon\left(q, q_{z}, \omega\right)$ for two $\mathrm{CuO}_{2}$ planes in a unit cell is

with,

$$
\varepsilon\left(q, q_{\mathrm{z}}, \omega\right)=1+2 P(q, \omega) S\left(q, q_{\mathrm{z}}\right)+P^{2}(q, \omega) S\left(q, q_{\mathrm{z}}\right) S^{\prime}(q),
$$

$$
S^{\prime}(q)=\frac{\cosh (q d)-\cosh \left(q d^{\prime}\right)}{\sinh (q d)},
$$

and $d^{\prime}=2 d_{1}-d$ with $d_{1}=d / 3$.

The layered structure superconductors possess anisotropic physical properties and as a matter of fact, it is customary to restrict ourselves to the $a-b$ conduction plane and for this purpose we average (2) over $q_{\mathrm{z}}$ as

$$
V(q, \omega)=\frac{d}{2 \pi} \int_{-\pi / \mathrm{d}}^{+\pi / \mathrm{d}} V\left(q, q_{\mathrm{z}}, \omega\right) \mathrm{d} q_{\mathrm{z}}
$$

The integral can thus be evaluated using (2)-(6) to get

Here

$$
V(q, \omega)=\frac{4 \pi e^{2}}{q \varepsilon_{0}} \frac{\left[1+P(q, \omega) S^{\prime}(q)+R(q, \omega)\right] \sin h(q d)}{\left[\left|D^{2}(q, \omega)-1\right|\right]^{1 / 2}} .
$$

with,

$$
R(q, \omega)=\frac{\sin h\left[q\left(d-d_{1}\right)\right]+D(q, \omega) \sin h\left(q d_{1}\right)}{\sin h(q d)},
$$

$$
D(q, \omega)=\cosh (q d)+2 P(q, \omega) \sin h(q d)+P^{2}(q, \omega) \sin h(q d) S^{\prime}(q)
$$

The polarization function $P(q, \omega)$ is due to the charge carriers (c) and ions (i)

$$
\begin{aligned}
P(q, \omega) & =P_{\mathrm{c}}(q, \omega)+P_{\mathrm{i}}(q, \omega) \\
& =\frac{2 \pi e^{2} n_{\mathrm{c}} q}{m^{*} \varepsilon_{\mathrm{o}}\left[\left(q^{2} V_{\mathrm{F}}^{2}\right) / 2-\omega^{2}\right]}-\frac{2 \pi e^{2} n_{\mathrm{i}} Z^{2} q}{M \varepsilon_{0} \omega^{2}},
\end{aligned}
$$

where $n_{\mathrm{c}}\left(n_{\mathrm{i}}\right)$ denotes the 2D charge carriers (ionic) density, $Z e$ the sum of ionic and bound electronic charge, $V_{\mathrm{F}}$ the Fermi velocity, $m^{*}$ the effective mass of the charge carriers and $M$ the mass of unit cell, respectively.

The interaction potential $V(q, \omega)$ properly incorporates the effect of electronelectron, electron-phonon and electron-plasmon interactions as well as the structure 
factor. The interaction energy is attractive if

$$
D(q,(b)<0,
$$

and is responsible for pairing mechanism. In the long wavelength limit $(q \rightarrow 0)$, the interaction potential leads to

with,

$$
V(q, \omega)=\frac{4 \pi e^{2} d}{\varepsilon_{0}} \varepsilon^{-1}(q, \omega)
$$

$$
\varepsilon^{-1}(q, \omega)=\frac{1+P(q, \omega) S^{\prime}(q)+R(q, \omega)}{\left[\left|D^{2}(q, \omega)-1\right|\right]^{1 / 2}} .
$$

Zero's of the dielectric function will yield the frequencies of coupled 2D acoustic plasmon and phonon modes. In the long wavelength limit, the frequencies are deduced as

$$
\begin{aligned}
& \omega_{+}^{2}=\$^{2}+\alpha \omega_{\mathrm{Pl}}^{2}, \\
& \omega_{-}^{2}=\alpha \omega_{\mathrm{Pi}}^{2}\left[1+\alpha \omega_{\mathrm{Pl}}^{2} / \$^{2}\right]^{-1} .
\end{aligned}
$$

Here, $\omega_{\mathrm{Pl}}\left(\omega_{\mathrm{Pi}}\right)$ are the usual $2 \mathrm{D}$ electron (ion) plasmon frequency, $\alpha=q d$ and $\$^{2}=q^{2} V_{\mathrm{F}}^{2} / 2$.

In order to calculate the superconducting transition temperature one requires the knowledge of modified Coulomb repulsive parameter $\left(\mu^{*}\right)$ and the coupling parameter $(\lambda)$ between neighbouring electrons. For this, the real and imaginary parts of the interaction potential is evaluated from (12) as

and

$$
\text { Real } V\left(q,(\omega)=\frac{4 \pi e^{2}}{q \varepsilon_{\theta}} \frac{\omega^{2}}{\left(\omega^{2}-C q\right)},\right.
$$

$$
\text { Imaginary } V\left(q .(\omega)=\frac{4 \pi e^{2}}{q \varepsilon_{0}} \pi C q \delta\left(\omega^{2}-C q\right)\right. \text {. }
$$

Here, $\left.\delta(\omega)^{2}-C q\right)$ is the Dirac delta function and $C=\omega_{\mathrm{Pi}}^{2} / q \varepsilon_{0}$. The Coulomb repulsive parameter $(\mu)$ is expressed as

$$
\begin{aligned}
\mu & =\frac{N(0)}{2 K_{F}^{2}} \int_{0}^{2 K_{r}} \operatorname{Real} V(q, \omega) q \mathrm{~d} q \\
& =\frac{2 \pi e^{2} \mathrm{~d}}{\varepsilon_{0}} N(0) \operatorname{Ln}\left[\frac{2+K}{K}\right],
\end{aligned}
$$

with $K=2 d / a_{\mathrm{B}}, N(0)$ as the density of states per unit cell and $a_{\mathrm{B}}$ is the Bohr radius.

Finally, the modified Coulomb repulsive parameter $\left(\mu^{*}\right)$ representing the electronelectron interaction for the maximum value of phonon frequency is

$$
\left.\mu^{*}=\frac{\mu}{1+\mu \operatorname{Ln}\left(E_{\mathrm{F}} / h(\omega)\right.}\right)
$$

with $E_{\mathrm{F}}$ as the Fermi energy and $\hbar \omega$.. denotes the $2 \mathrm{D}$ acoustic phonon energy. 
The electron-phonon coupling parameter $(\lambda)$ is derived from the Eliashberg function $\alpha^{2}(\omega) F(\omega)$ through (Eliashberg 1960$)$

$$
\lambda=2 \int_{0}^{\omega-} \frac{\alpha^{2}(\omega) F(\omega)}{\omega} \mathrm{d} \omega,
$$

with $F(\omega)$ as the phonon density of state and $\alpha^{2}(\omega)$ as coupling strength between electrons and phonons. The coupling parameter is deduced from the Imag. $V(q, \omega)$ as

$$
\begin{aligned}
\alpha^{2}(\omega) F(\omega) & =\frac{N(0)}{2 K_{\mathrm{F}}^{2}} \int_{0}^{2 K_{\mathrm{F}}} \text { Imag. } V(q, \omega) q \mathrm{~d} q \\
& =\frac{N(0) \pi^{2} e^{2} \omega^{2} \mathrm{~d}}{\varepsilon_{0} C K_{\mathrm{F}}^{2}\left(1-\omega_{-}\right)^{1 / 2}} .
\end{aligned}
$$

Thus, the electron-phonon coupling parameter is obtained as

$$
\lambda=\frac{\omega_{-}^{2}}{2 a_{\mathrm{B}} K_{\mathrm{F}}^{2} C} .
$$

Using the above expression for $\lambda, \mu^{*}$ and $\omega_{-}$, the superconducting transition temperature $\left(T_{\mathfrak{c}}\right)$ due to phonons alone is (Ruvalds 1987)

$$
T_{\mathrm{c}}^{\mathrm{Ph}}=0 \cdot 7 \omega_{-} \exp \left[-\frac{1+\lambda}{\lambda-\mu^{*}}\right] .
$$

When one considers the presence of both 2D acoustic phonons and plasmons, the total $T_{\mathrm{c}}$ is $(\mathrm{Kr}$ resin 1987)

where

$$
T_{\mathrm{c}}=T_{\mathrm{c}}^{\mathrm{Ph}}\left[\frac{\hbar \omega_{\mathrm{P} 1}}{T_{\mathrm{c}}^{\mathrm{Ph}}}\right]^{h},
$$

$$
h=\frac{\lambda_{\mathrm{Pl}}}{\lambda+\lambda_{\mathrm{P} 1}} .
$$

$\lambda_{\mathrm{P} 1}$ denotes the electron-plasmon coupling strength.

Using the developed expressions following the strong coupling theory, we have obtained the coupling parameters $\left(\lambda\right.$ and $\left.\mu^{*}\right)$ and $2 \mathrm{D}$ acoustic phonon (plasmon) modes as a function of oxygen deficiency to compute the variation of superconducting transition temperature with oxygen deficiency in $\mathrm{ABa}_{2} \mathrm{Cu}_{3} \mathrm{O}_{7-\delta}$ superconductors. The analysis is presented and discussed in the following section.

\section{Results and discussion}

In order to calculate the superconducting transition temperature $\left(T_{\mathrm{c}}\right)$ in $\mathrm{ABa}_{2} \mathrm{Cu}_{3} \mathrm{O}_{7-\delta}(\mathrm{A}=\mathrm{Y}, \mathrm{Yb})$ superconductors, we have used the realistic parameters based on the experimental data. The effective mass is evaluated from the electronic specific heat coefficient $(\gamma)$ value as $1.5 m_{\mathrm{e}}$ for $\mathrm{YBa}_{2} \mathrm{Cu}_{3} \mathrm{O}_{7-\delta}$ superconductors (Inderhees et al 1987). For $\mathrm{YbBa}_{2} \mathrm{Cu}_{3} \mathrm{O}_{7-\delta}$ system, we used $m^{*}=1.6 m_{\mathrm{e}}$, although no 

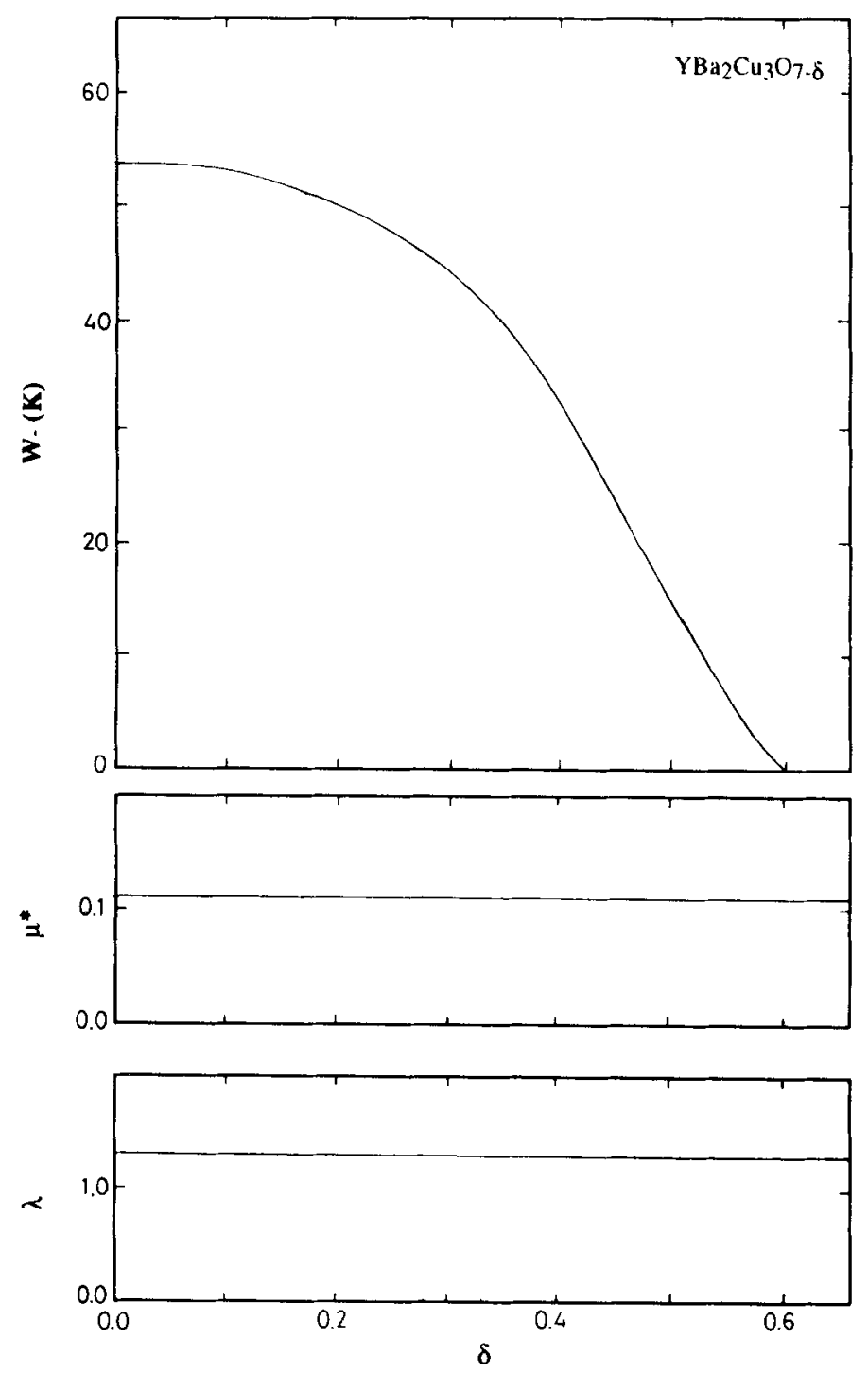

Figure 2. Variation of $\omega_{-}(K), \mu^{*}$ and $\lambda$ with $\delta$ in $\mathrm{YBa}_{2} \mathrm{Cu}_{3} \mathrm{O}_{7-\delta}$ superconductors.

experimental data on electronic specific heat coefficient is available, so we choose it accordingly. The mass of a unit cell is 12.77 amu and the background dielectric constant $\varepsilon_{0}$ is taken as 4.5 (Bozovic 1990) from the electron energy loss spectroscopy. The charge carrier density and ionic density are evaluated from the lattice parameters. Taking $a=3.8136 \AA, b=3.8845 \AA$ and $c=11.6603 \AA$ (Cava et al 1990) we obtained $n_{\mathrm{c}}=2.38 \times 10^{14} \mathrm{~cm}^{-2}$ and $n_{\mathrm{i}}=6.74 \times 10^{14} \mathrm{~cm}^{-2}$ at $\delta=0.0$. It is customary to make them oxygen deficient $(\delta)$ dependence as

$$
n_{\mathrm{c}(\mathrm{i})}(\delta)=n_{\mathrm{c}(\mathbf{i})}(\delta=0 \cdot 0)\left[\exp -\left(\delta / \delta_{\mathrm{c}}\right)^{2}\right]
$$

We choose $\delta_{\mathrm{c}}=0 \cdot 4$. The sum of ionic and bound electronic charge $Z e=-2 e$. 

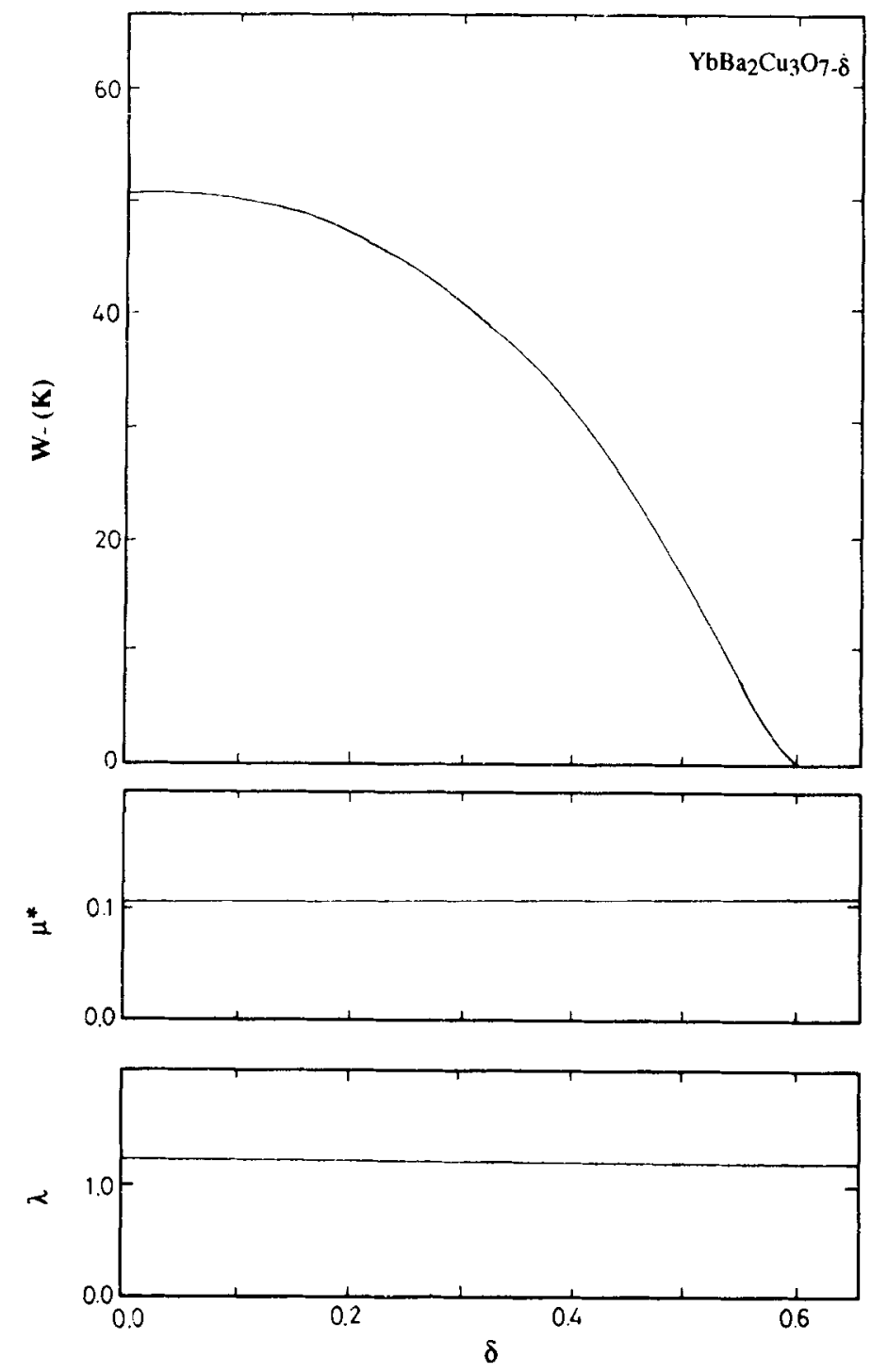

Figure 3. Variation of $\omega(K), \mu^{*}$ and $\lambda$ with $\delta$ in $\mathrm{YbBa}_{2} \mathrm{Cu}_{3} \mathrm{O}_{7-\delta}$ superconductors.

The coupling parameters ( $\lambda$ and $\mu^{*}$ ) and the 2D acoustic phonon energy ( $\left.\hbar \omega_{-}\right)$as discussed in previous section have been plotted as a function of $\delta$ in figures 2 and 3 for $\mathrm{YBa}_{2} \mathrm{Cu}_{3} \mathrm{O}_{7-\delta}$ and $\mathrm{YbBa}_{2} \mathrm{Cu}_{3} \mathrm{O}_{7-\delta}$ superconductors, respectively. The electronphonon coupling parameter $(\lambda)$ is deduced as $1.28(1.20)$ for $\mathrm{Y}(\mathrm{Yb})$, respectively. It is seen from the figures that $\lambda$ remains almost constant for the whole oxygen deficiency range. The estimated value of $\lambda>1.0$ favours the strong coupling theory. The screened Coulomb repulsive parameter $\mu^{*}$ is obtained as $0.11(0.10)$ for $\mathrm{Y}(\mathrm{Yb})$ cuprate superconductors and its behaviour with $\delta$ is almost constant. The $2 \mathrm{D}$ acoustic phonon energy is obtained as $46(42) \mathrm{meV}$ for $\mathrm{Y}(\mathrm{Yb})$. This is in accordance with the previous Raman measurements (Thomsen and Cardona 1989). It is clear from the figure that 


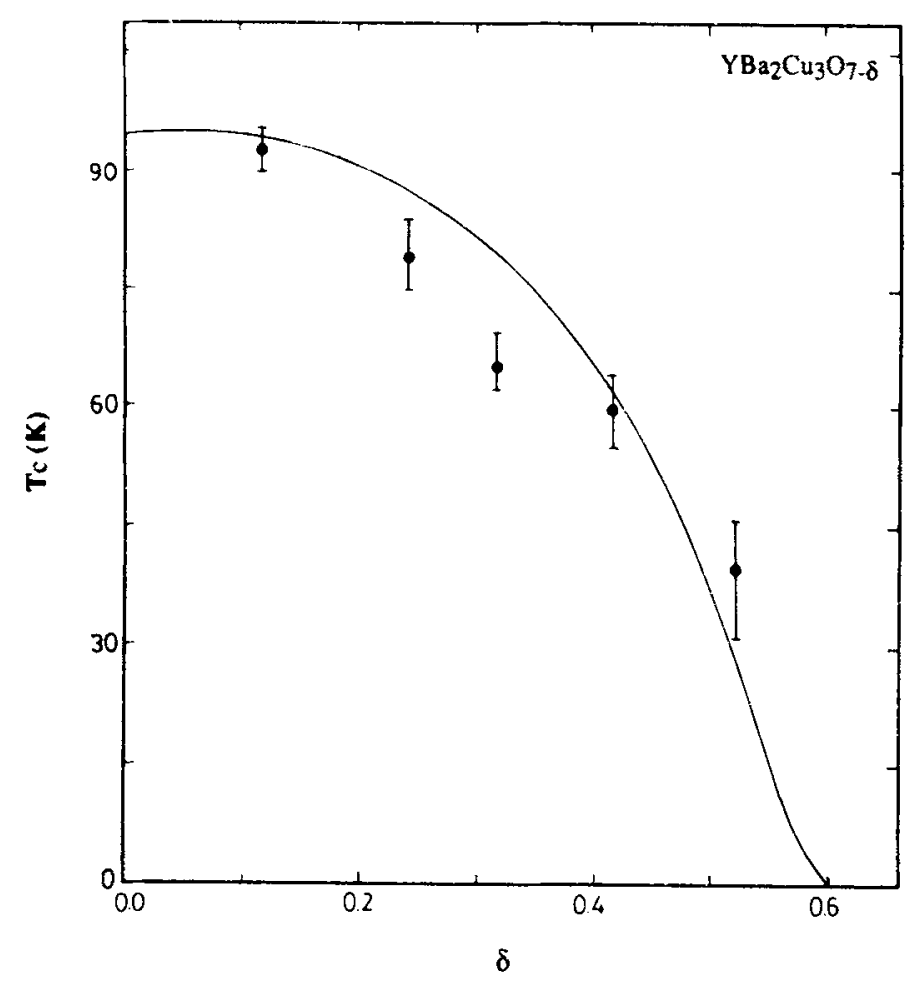

Figure 4. Variation of $T_{\mathrm{c}}$ with $\delta$ in $\mathrm{YBa}_{2} \mathrm{Cu}_{3} \mathrm{O}_{7-\delta}$ superconductors. Experimental data $(\boldsymbol{\phi})$ are taken from Kerkels et al (1992).

$\omega_{-}$decreases with the increased value of $\delta$. We believed that for the higher values of oxygen deficiency, charge carriers participating in the pairing mechanism is reduced and hence the low density of states. If we consider only phonon mechanism the transition temperature estimated from (22) comes out to be $53 \cdot 21(57 \cdot 00) \mathrm{K}$ for $\mathrm{Y}(\mathrm{Yb})$ doped superconductors. The $T_{\mathrm{c}}$ obtained by us with the phonon mechanism is quite low as compared to the experimental data. This motivated us to incorporate the $2 \mathrm{D}$ acoustic plasmons. Taking $\hat{\lambda}_{\mathrm{PI}}=0.1$ and $h\left(\omega_{+}\right.$as $3 \mathrm{eV}$ from (13), we find that $T_{\mathrm{c}}$ enhances to $95 \mathrm{~K}$ which is consistent with the reported value of $T_{\mathrm{c}}=90 \mathrm{~K}$. Hence, there is an enhancement of $78 \%$ on $T_{\mathrm{c}}$ values as obtained from the phonon mechanism. For the $\mathrm{YbBa}_{2} \mathrm{Cu}_{3} \mathrm{O}_{7-\delta}$ superconductors, we obtained $92 \mathrm{~K}$ which is in fair agreement with the reported value of $T_{c}=89 \mathrm{~K}$.

We have made efforts to understand the behaviour of $T_{\mathrm{c}}$ with $\delta$ in $\mathrm{Y}(\mathrm{Yb}) \mathrm{Ba}_{2} \mathrm{Cu}_{3} \mathrm{O}_{7-\delta}$ superconductors and is shown in figures 4 and 5 . From the proposed theory with $2 \mathrm{D}$ conducting $\mathrm{CuO}_{2}$ layers in a unit cell we obtained the variation of $T_{\mathrm{c}}$ with $\delta$ similar to those reported earlier. It is found that with the increase of $\delta$ the $T_{\mathrm{c}}$ drops linearly and is zero for $\delta=0 \cdot 6$. Furthermore, the theory fails to predict the second plateau which occurs in the range $0.3<\delta<0.5$. We believe that the second plateau might be due to the phase transition occurring in this region. The deduced results are consistent with the reported reports and is attributed to the proper care of layered structure with two conducting $\mathrm{CuO}_{2}$ planes in a unit cell of yttrium cuprates. 


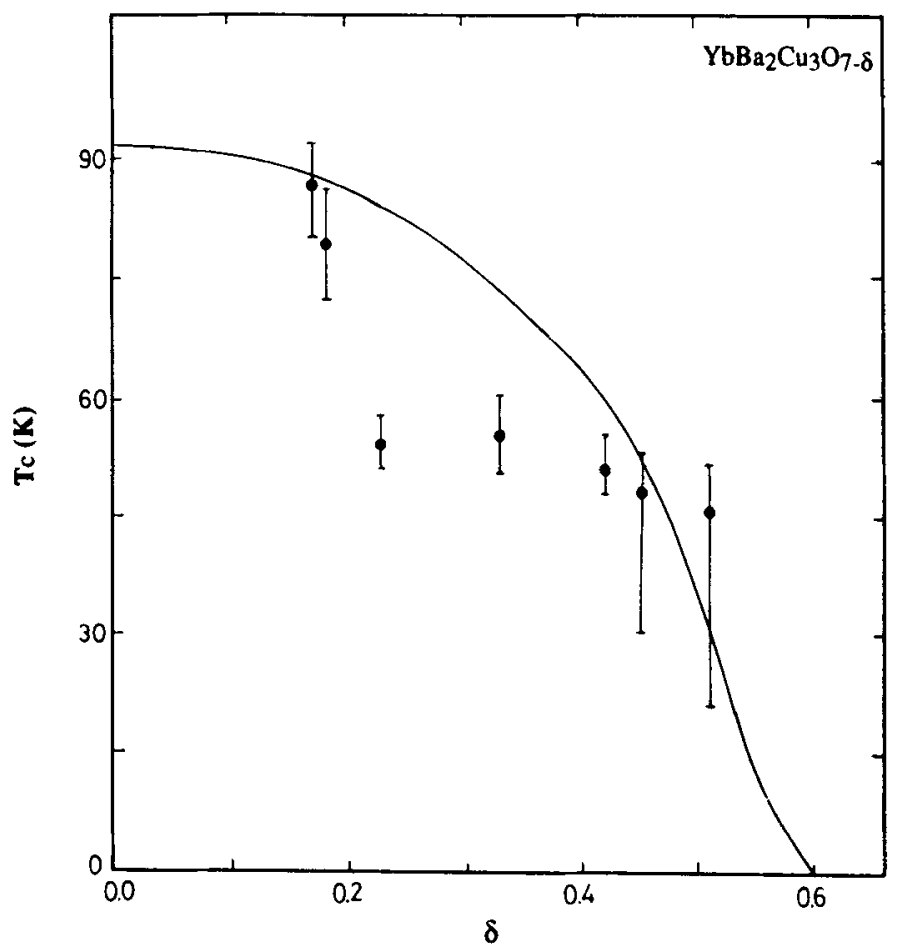

Figure 5. Variation of $T_{\mathrm{c}}$ with $\delta$ in $\mathrm{YbBa}_{2} \mathrm{Cu}_{3} \mathrm{O}_{7-\delta}$ superconductors. Experimental data (\$) are taken from Kerkels et al (1992).

\section{Conclusions}

In the present investigation, we have systematically investigated the pairing mechanism in yttrium cuprates by considering two conducting $\mathrm{CuO}_{2}$ planes in a unit cell. The model potential properly incorporates the electron-electron, electron-phonon and electron-plasmon interactions. Deduced value of $2 \mathrm{D}$ acoustic phonon and plasmon modes are consistent with the Raman measurements and electron energy loss spectroscopy in yttrium cuprates. The coupling parameters as $\lambda$ and $\mu^{*}$ from the above approach favours the strong coupling theory. It is noticed that the role of $2 \mathrm{D}$ acoustic plasmons is to provide an additional pairing mechanism that could lead to such high $T_{\mathrm{c}}$ values. We are able to understand the first plateau observed in the variation of $T_{\mathrm{c}}$ with $\delta$. The obtained variation is quite consistent with the experimental data. Regarding the second plateau observed at $T_{\mathrm{c}}$ of $60 \mathrm{~K}$ we feel that this is due to the phase transition and will be studied separately in future. In conclusion, the $2 \mathrm{D}$ acoustic phonons together with $2 \mathrm{D}$ acoustic plasmons can explain fairly well the pairing mechanism and the superconducting transition temperature in yttrium cuprate superconductors.

\section{Acknowledgement}

The authors are thankful to the University Grants Commission (UGC), New Delhi for financial support. 


\section{References}

Bednorz J G and Muller K A 1986 Z. Phls. B64 189

Bozovic I 1990 Phys. Ret. B42 1969

Cava R S et al 1990 Physica C165 419

Eliashberg G M 1960 Sol. Phy (JETPI 11096

Inderhees S E, Salamon M B. Friedmann T A and Ginsberg D M 1987 Phys. Ret. B36 2401

Jha S S 1987 Pramana J. Phys. 29 L615

Kerkels T, Zou H, Ven Tendeloo G, Wagener D, Buchgeister M, Hosseini S M and Herzog P 1992 Physica C196 363

Kresin V Z 1987 Phys. Rev. B35 3116

Ruvalds J 1987 Phy's. Rev. B35 8869

Singh R K. Varshney D. Gaur N K and Sharma A C 1993 Ball. Mater. Sci. 16117

Tewari S P and Gumber P K 1990 Physica C171 147

Thomson C and Cardona M 1989 in Physicat properties of high temperature superconductors (ed.) D M Ginsberg (Singapore: World Scientific) Vol. 1 p. 409

Varshriey D and Singh R K 1995 Phys. Rer B52 7629

Wu M K et al 1987 Phis. Ret. Litt. $\mathbf{5 8} 908$ 